\title{
Millimetre/Sub-millimetre Observations of Circumstellar Disks
}

\author{
Anne Dutrey ${ }^{1}$ \\ ${ }^{1}$ Laboratoire d'Astrophysique de Bordeaux, OASU, CNRS UMR 5804, 2 rue de l'observatoire, \\ BP89, F-33271 Floirac Cedex, France \\ email: Anne.Dutrey@obs.u-bordeaux1.fr
}

\begin{abstract}
TTauri disks located in nearby star-forming regions (e.g. Taurus-Auriga at $140 \mathrm{pc}$ ) are thought to be the site of planet formation, since proto-planetary disks orbiting around active (still accreting) TTauri stars should contain, in many cases, enough gas to form giant gaseous planets. As such, circumstellar disks are ideal laboratories to study planet formation, provided the gas and dust observations have enough sensitivity and resolving power. I will focus in these proceedings, on recent results of molecular observations which unveil the physical conditions of gas disks and reveal the weakness of our current understanding and modeling.
\end{abstract}

Keywords. astrochemistry, stars: formation, planetary systems: protoplanetary disks

\section{Introduction}

Since the early nineties, optical, near infrared (NIR) and millimetre/submillimetre ( $\mathrm{mm} / \mathrm{submm})$ observations of low and intermediate mass stars ( $\sim 1 \mathrm{Myr})$, such as TTauri and Herbig Ae stars, have shown that these young stars are surrounded by circumstellar disks. These proto-planetary disks, residuals from the molecular cores which have formed the stars, still contain enough gas and dust to form planets. Although the dust emission in the optical and in NIR (scattered light emission) is the easiest tracer of proto-planetary disks, $\mathrm{H}_{2}$ molecules (which does not deplete on dust grains) represent more than $70 \%$ of the total mass in disks and remain difficult to observe because of the lack of a permanent dipole moment. Some direct $\mathrm{H}_{2}$ detections are possible but the observed lines mainly trace the warm gas located in the inner disks $(\mathrm{R}<10-20 \mathrm{AU})$, while in many cases, disks are known to extend out to several $100 \mathrm{AU}$. These cool $(\sim 10-50 \mathrm{~K})$ outer regions, which may even contain most of the mass, can only be characterized by molecules having rotational lines from low energy levels and detectable with large millimeter and sub-millimeter interferometers. CO is the most abundant molecule (even if it can deplete on dust grains) after $\mathrm{H}_{2}$ and its lowest rotational lines are easily excited (and thermalized) by collisions with $\mathrm{H}_{2}$. Current heterodyne $\mathrm{mm} / \mathrm{submm}$ arrays begin to reach the sensitivity, resolving power and spectral resolution needed to map cold molecular disks and millimeter spectro-imaging have revealed, for example, that disks are in Keplerian rotation. The SMA has recently opened the sub-millimeter window, while the IRAM Plateau de Bure interferomter $(\mathrm{PdBI})$ routinely provides images of 0.6 " resolution at $1.3 \mathrm{~mm}$. Therefore, recent $\mathrm{mm} / \mathrm{submm}$ observations have provided many new direct constraints on physical and chemical structure of disks which could not be addressed by disk continuum observations. With ALMA entering in operation, our current knowledge on planet forming regions in disks will likely significantly improve in the next years.

In this review, I will focus on recent molecular results which provide new constraints on the gas chemistry and physics in disks. In particular, I will present studies obtained with the IRAM array in the context of the CID ("Chemistry in Disks") project, a 
European collaboration dealing with the observation and modeling of gas disks to retrieve the physical and chemical conditions leading to planet formation. $\dagger$ After a general presentation of the state of art of molecular gas disks, I will present our new results and discuss how they can change our current thinking about disk chemistry.

\section{Current Model for Gas and Dust Disks}

For disks of about 1 Myr old, the mass and dynamics are dominated by the gas and the gas-to-dust ratio is supposed to be of the order of 100 but there is no accurate measurements of this quantity yet. Another major unknown in disks is the exact radial and vertical distribution of the gas and its evolution with time. This is particularly critical in the inner regions $(\mathrm{R}<20-50 \mathrm{AU})$ where planet formation should occur.

So far, molecular surveys are sensitivity limited to the most abundant molecules, namely those found in cold molecular clouds. In addition to $\mathrm{CO},{ }^{13} \mathrm{CO}$ and $\mathrm{C}^{18} \mathrm{O}$, only $\mathrm{HCO}^{+}, \mathrm{H}^{13} \mathrm{CO}^{+}, \mathrm{DCO}^{+}, \mathrm{CS}, \mathrm{HCN}, \mathrm{HNC}, \mathrm{DCN}, \mathrm{CN}, \mathrm{H}_{2} \mathrm{CO}, \mathrm{N}_{2} \mathrm{H}^{+}$and $\mathrm{C}_{2} \mathrm{H}$ have been firmly detected. The study of these molecules can however improve our knowledege on the gas disk density provided the excitation conditions are properly understood and modeled (Dutrey et al. (1997)). As soon as the full ALMA array will be operational, the detection rate of molecules may significantly increase, revealing the complexity of the disk molecular chemistry and its vertical stratification.

\subsection{Current Gas Disk Structure}

Chemistry is an essential agent in the shaping of the gas disks. Molecular (and atomic) abundances also play a key role in determining the cooling rate of the gas, and thereby the thermal balance of the disk. It is then essential to understand where in the disk the molecules are located.

With the exception of the very inner disk $(R \leqslant 10 \mathrm{AU})$ where accretion can be a source of heat, disks are mostly heated by the stellar UV radiation and X rays, the interstellar UV field and the cosmic rays. This leads to the current scheme where both the gas and dust have vertical and radial temperature variations, the upper layers being superheated by the stellar UV field (Chiang \& Goldreich (1997)). Given this temperature and the vertical and radial density distributions (the gas is expected to be in hydrostatic equilibrium), one can vertically defines three different "chemical" zones. The disk upper layer acts like a PDR formed of ions and then neutral gas, deeper in the disk, there is the warm molecular layer and then the cold mid-plane, mostly devoid of molecules, with the exception of $\mathrm{H}_{2}$. The mid-plane temperature drops significantly below the freeze out temperature of $\mathrm{CO}$, with a chemistry close to that of cold dense cores. This standard model is illustrated in Fig.1.

\subsection{Measuring temperature and densities from molecular lines Observations}

An important source of uncertainty in the analysis of molecular maps comes from the existence of non-LTE effects in the line excitation. A proper analysis of any line detection in disks requires then adequate handling of the line formation process, and in particular of the disk kinematics. Failure to do so can result in mis-interpretation of spectral lines, as shown by Guilloteau et al. (2006) for HDO in DM Tau. Modeling the surface density and temperature are usually done assuming power law radial dependencies with in some cases an exponential decay to express the viscous spread out of the surface density (see for example, Guilloteau et al. (2011)) .

$\dagger$ The CID consortium is an international collaboration (Dutrey et al. 2007b) between researchers from IRAM, MPIA and LAB which have been recently extended to american and taiwanese collaborators. 
Fortunately, the first levels of the CO rotational lines $(\mathrm{J}=2-1$ and $\mathrm{J}=1-0)$ are thermalized and hence allow a direct measurement of the gas temperature and surface density depending on the line opacity. For a thermalized transition, the brightness temperature $T_{B}(r)$ which is measured by the interferometer, is $\sim T_{K}$ (kinetic temperature) in the optically thick case and is a function of $\Sigma(r), T_{K}(r)(\Sigma(r)$ being the surface density) depending on the transition for a partially optically thin line. It roughly scales as $\Sigma(r) / T_{K}(r)$ at high temperatures (see Dutrey et al. (2007a) for a discussion).

Due to different opacities, the ${ }^{12} \mathrm{CO},{ }^{13} \mathrm{CO} \mathrm{J}=1-0$ and $\mathrm{J}=2-1$ sample different disk layers. In the case of DM Tau, the pioneer CO study of Dartois et al. (2003) confirmed predictions from models of disks externally heated by their central star (e.g. D'Alessio et al. (1999)). Close to the mid-plane, the temperature appears significantly cooler $(\sim$ $13 \mathrm{~K})$ than at the $\mathrm{CO}$ disk surface $(\sim 30 \mathrm{~K}$ at $100 \mathrm{AU})$. This happens in the region of the disk where the dust is still optically thick to the stellar radiation while it is already optically thin to its own emission, around $r \sim 50-200$ AU in the DM Tau case. Beyond $r \geqslant 200$ AU where the dust becomes optically thin to both processes, the temperature profile appears vertically isothermal (Dartois et al. (2003)). The fact that a significant fraction of the DM Tau disk has a temperature apparently below the CO freeze out point $(17 \mathrm{~K})$ has been interpreted in the framework of the turbulence where vertical mixing should mix the vertical molecular layers. This result also suggests that photo-desorption rates are not properly known (Hersant et al. (2009)).

Using the IRAM array, Pietu et al. (2007) refine the study of DM Tau by measuring both the temperature and the surface density of $\mathrm{CO}$ and $\mathrm{HCO}^{+}$in $\mathrm{DM}$ Tau, LkCa15 and MWC480. Fig. 2 shows the results of the $\chi^{2}$ minimization performed on the resolved observations (LTE conditions are reached for the observed transitions) in DM Tau. The right scale presents the signal-to-noise ratio obtained on the minimized parameter versus radius. At high opacity (e.g. ${ }^{12} \mathrm{CO} \mathrm{J}=2-1$ ), the temperature can be measured in a significant disk fraction while the estimate of the molecular surface density is only possible in the outer optically thin region. This is the opposite for an almost optically thin

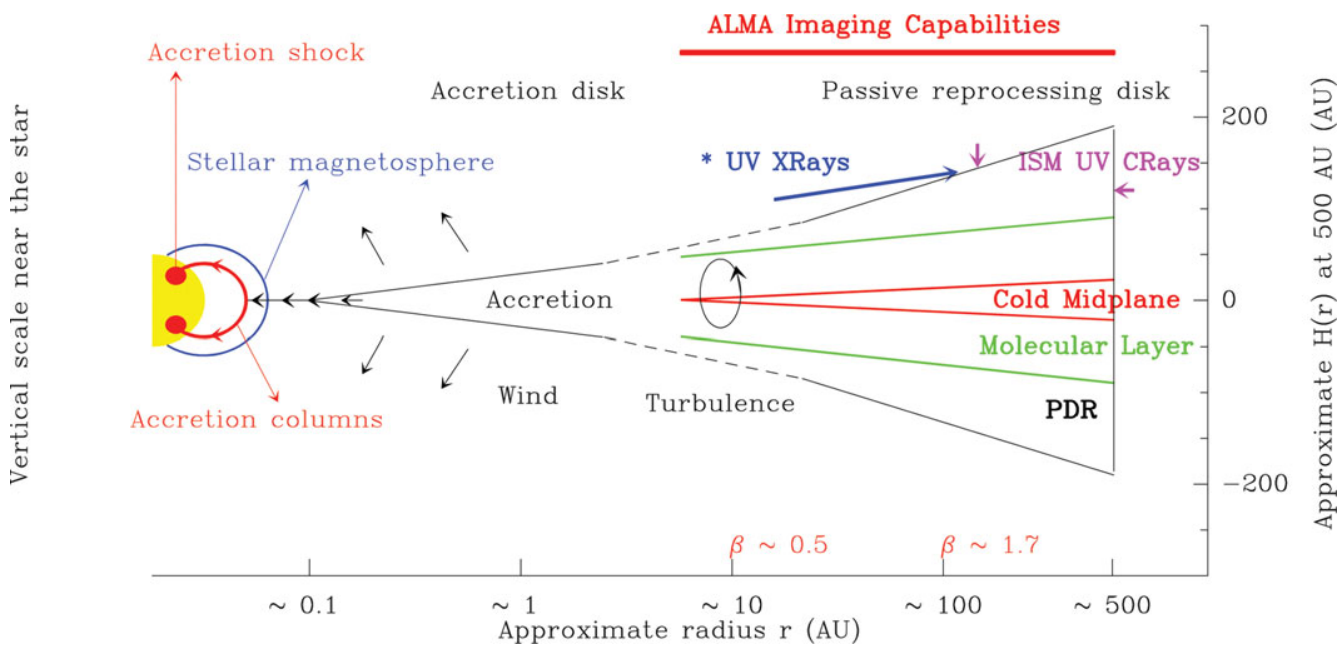

Figure 1. A schematic view of a protoplanetary disk orbiting a TTauri star and located in a nearby star-forming region $(150 \mathrm{pc})$. Current models predict three layers from the mid-plane to the top: 1$)$ the cold mid-plane $(\sim 10 \mathrm{~K})$ where most molecules are frozen on grains, 2$)$ the intermediate warm $(\sim 20-40 \mathrm{~K})$ molecular layer and 3$)$ the Photon dominated (PDR) upper layer. Following the recent results from Guilloteau et al. (2011), the expected value of the dust spectral index $\beta$ versus radius in the mm range is given. 
transition such as ${ }^{13} \mathrm{CO} \mathrm{J}=1-0$. This method will remain robust using ALMA and the exceptional sensitivity and resolving power of the new array will also allow observers to refine the accuracy on the radial and vertical dependencies of the temperature and density. Moreover, such a method can also be extended to higher CO transitions which would sample the disk atmosphere revealing the impact of X-rays and UV irradiation on the disk (Qi et al. (2006)).

Finally since $\mathrm{CO}$ observations are usually not sensitivity limited, spectro-imaging of $\mathrm{CO}$ provides invaluable information on the disk structure and geometry. In a recent paper, Dutrey et al. (2008) have found that the Keplerian gas disk of GM Aur was innerly truncated at $\sim 20 \mathrm{AU}$ by studying the $\mathrm{CO}$ velocity pattern observed with the IRAM array. In a Keplerian velocity field, the presence of an inner cavity of radius $r_{i n}$ would suppress line emission at velocities above $V_{r_{i n}}=\sqrt{\frac{G \cdot M_{*}}{r_{i n}}} \cdot \sin (i)$ compared to the systemic velocity ( $i$ being the inclination angle). Such an analysis is only possible on data with both high spectroscopic resolution (e.g. $0.1 \mathrm{~km} / \mathrm{s}$ ) and signal-to-noise ratio. This will likely become very common using ALMA to study the gas content of inner disks and constrain photo-evaporation processes or possible tidal interactions in the planet forming zone.
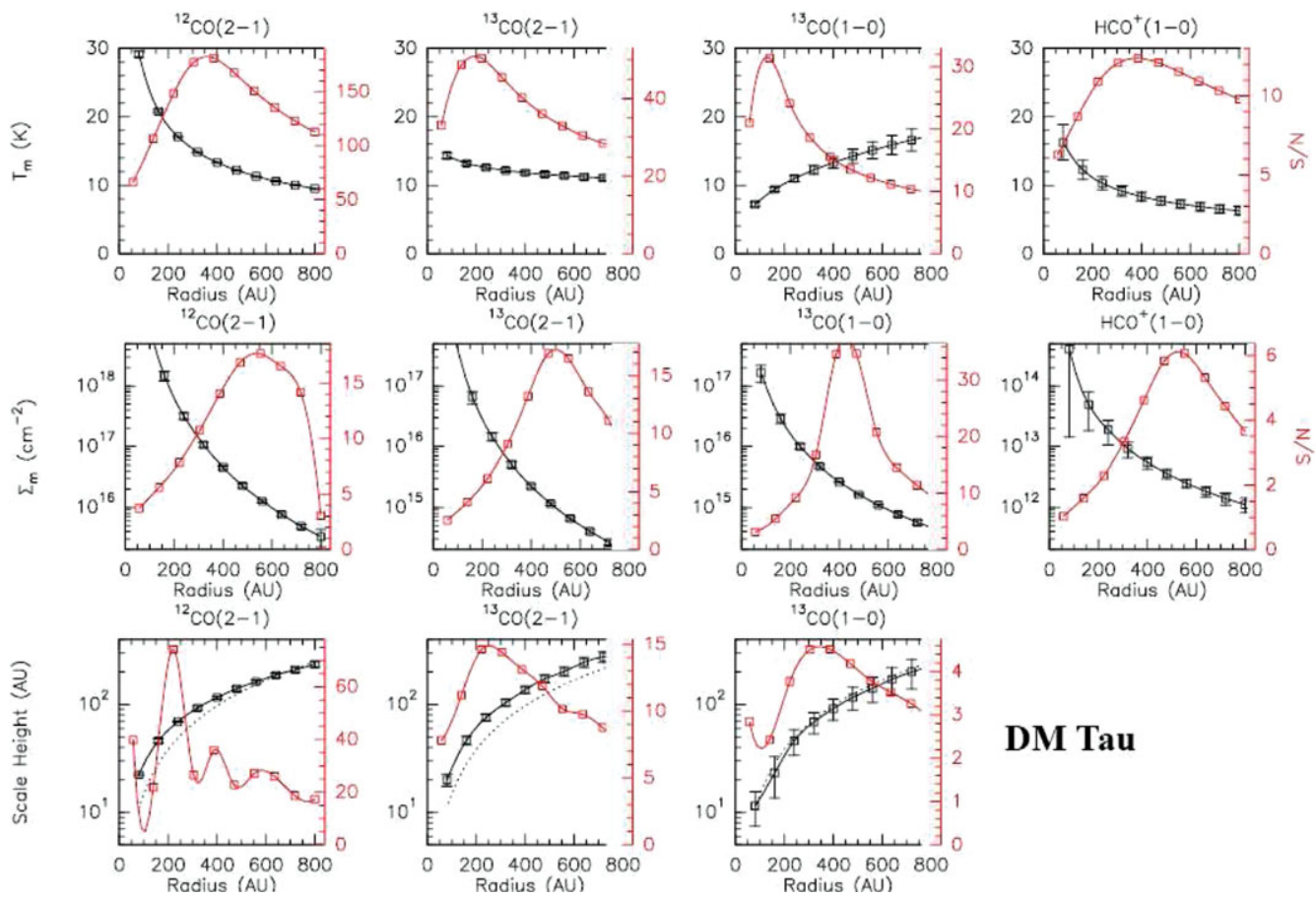

\section{DM Tau}

Figure 2. From Pietu et al. (2007). Analysis of IRAM PdBI observations of the DM Tau gas disk. Top panel: Kinetic temperature versus radius, Medium panel: Molecular surface densities versus radius, Top panel: scale height versus radius. These physical parameters are respectively derived from left to right (by decreasing opacity): ${ }^{12} \mathrm{CO} \mathrm{J}=2-1,{ }^{13} \mathrm{CO} \mathrm{J}=2-1,{ }^{13} \mathrm{CO} \mathrm{J}=1-0$ and $\mathrm{HCO}^{+} \mathrm{J}=1-0$. The black scale corresponds to the parameter scale, the red one to the $\mathrm{S} / \mathrm{N}$ ratio on the derived parameter. The kinetic temperature is better determined in the inner part of the disk which is optically thick, while the surface density is better determined in the optically thin outer part. The scale height is more difficult to constrain but $\mathrm{CO}$ results are in agrement with an hydrostatic regime. 


\section{New Results derived from recent $\mathrm{mm} /$ submm molecular Surveys}

Fig.3 illustrates the quality of data which is now obtained with current arrays. Such a quality is required to properly measured physical gas disk parameters and begin quantitative comparisons with surface density predictions from chemical models.

\subsection{Global Properties}

In the recent years, spectro-imaging of molecular disks have also open to investigations some important ingredients which play a role both in the chemistry and in the physics of disks. Current arrays allow observers to derive averaged values on the whole disk but ALMA will provide in many cases radial dependencies on these key parameters.

\subsubsection{Turbulence}

Most measurements of the turbulence in disks are indirect and rely on the $\alpha$ prescription through modeling of the spectral energy distribution (SED), or radial density profile (Guilloteau et al. (2011)). Dartois et al. (2003) then Pietu et al. (2007) made the first direct measurement of the random component of the gas velocity field from spatially and spectrally resolved molecular observations. Their analysis of the CO maps on DM Tau and LkCa15 revealed that in outer TTauri disks the averaged value of the turbulence correspond to largely subsonic motions.

\subsubsection{Ionization}

Only a few molecular ions have been observed in disks: $\mathrm{HCO}^{+}, \mathrm{DCO}^{+}$and $\mathrm{N}_{2} \mathrm{H}^{+}$(Pietu et al. (2007), Dutrey et al. (2007b), Qi et al. 2008). The most abundant molecular ion is $\mathrm{HCO}^{+}$. Dutrey et al. $(2007 \mathrm{~b})$ found a $\mathrm{N}_{2} \mathrm{H}^{+} / \mathrm{HCO}^{+}$ratio of $\sim 0.02-0.05$ in the DM Tau and LkCa15 disks, a value similar to that found in cold dark clouds. Deriving an ionization fraction from the current data remain delicate. Using $\mathrm{CO}$ and $\mathrm{HCO}^{+}$observations from the SMA, Qi et al. (2008) have reported an ionization fraction of $X\left(e^{-}\right)=n\left(e^{-}\right) / n\left(H_{2}\right) \simeq$ $10^{-7}$ in TW Hydra.

\subsubsection{Deuteration}

Since $\mathrm{H}_{2} \mathrm{D}^{+}$is not yet detected, $\mathrm{DCO}^{+}$and DCN remain the only deuterated molecular species observed in disks (e.g. DM Tau: Guilloteau et al. (2006) and TW Hydra: Qi et al. (2008)). Searching for deuterated species in disks clearly requests sensitivity and ALMA (Chapillon et al. (2011a)).

\subsection{The layered chemical Model}

\subsubsection{Towards a colder molecular layer?}

After CO, other molecules such as CCH (Henning et al. (2010)) or CN and HCN (Chapillon et al. 2011b) have been studied in DM Tau, LkCa15 and MWC 480. In all cases, the chemical modeling reproduces the magnitude of the observed column densities within a factor 3-10 but fails to reproduce its radial dependencies. Surprisingly, the analysis of the DM Tau and LkCa15 disks gives excitation temperatures around $10 \mathrm{~K}$ or even below $(\sim 6 \mathrm{~K})$ beyond a radius of 100 AU. For MWC480, Chapillon et al. 2011 have derived from their study, an excitation temperature for the CN J=2-1 line which is around $30 \mathrm{~K}$, in agreement both with the kinetic temperature derived by Pietu et al. (2007) from their CO analysis, and chemical predictions for the molecular layer. Since MWC480 is an Herbig Ae star of spectral type A4, the disk is also expected to be warmer and molecules may remain in the gas phase. 


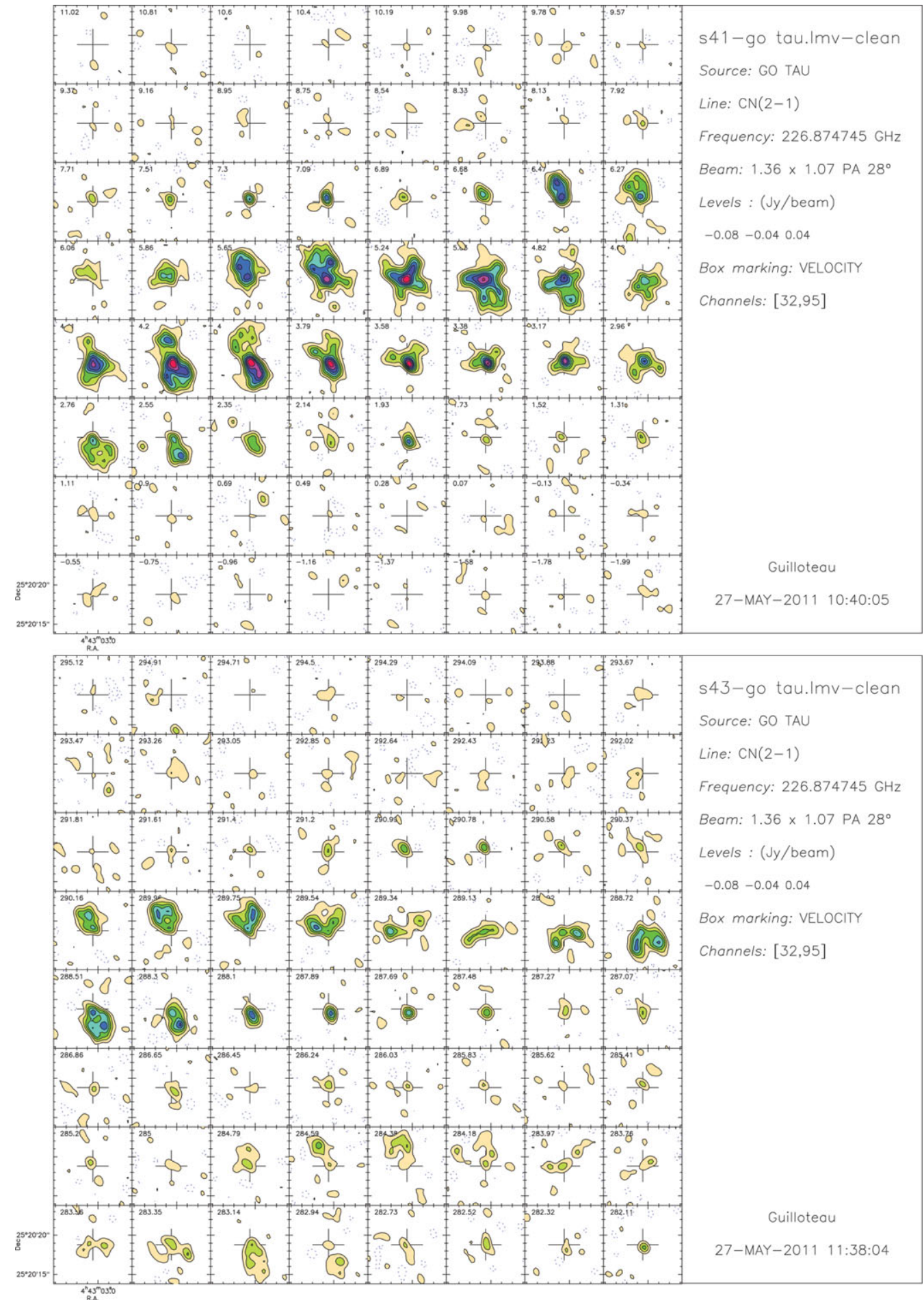

Figure 3. Example of observations obtained from arrays such as the IRAM interferometer. CN $\mathrm{J}=2-1$ in the disk surrounding the TTauri star GO Tau. Top: several hyperfine components, Bottom: the main group. The spectral resolution is $0.2 \mathrm{~km} / \mathrm{s}$ and the angular resolution is $\sim 1.2^{\prime \prime}$. From Guilloteau et al. (2012), in prep. This illustrates the typical quality requested to derive the gas disk properties. These observations have been obtained in the context of the CID ("Chemistry In Disks") international collaboration. 
To explain the apparently low temperatures observed in the TTauri disks, several possibilities can be invoked. First of all, using expected physical conditions, the observed transitions ( $\mathrm{CCH}$ 1-0, 2-1, HCN 1-0 and CN 2-1) should be thermalized at least in a large fraction of these disks. Furthermore sub-thermal excitation is not sufficient to explain these low temperatures which are also similar to those derived from thermalized CO J-0 and $\mathrm{J}=2-1$ transitions (Pietu et al. (2007)). Turbulence may also play a role by allowing vertical mixing from warmer upper molecular layers (Semenov et al. (2006), Aikawa (2007)). In the case of DM Tau, Chapillon et al. 2011b have investigated the possibility to have a lower gas-to-dust ratio (by about a factor $\sim 6$ ). In that case sub-thermal excitation remains possible but the predicted column densities from $\mathrm{HCN}$ and $\mathrm{CN}$ are low compared to the observed ones. Finally, another possibility would be that "cold" chemistry for molecules such as $\mathrm{CN}$ or $\mathrm{CCH}$ and in particular photo-desorption rates are poorly known (Öberg et al. (2009), Hersant et al. (2009)).

Waiting from more accurate observations with ALMA, these apparently low temperatures point toward a colder molecular layer. This strongly suggests that our current knowledge of the disk physics (thermal and density structure, role of dust settling, gasto-dust-ratio estimate, vertical and radial mixing, etc... ) and chemistry is more limited than commonly believed.

\subsubsection{Characterization of the Mid-Plane}

Tracing the cold mid-plane is challenging since most molecules should be frozen onto grains. The best candidate to trace this depleted zone is the ion $\mathrm{H}_{2} \mathrm{D}^{+}$because it is formed in gas phase at low temperature and in a so cold environment, it cannot be chemically destroyed by molecules such as $\mathrm{N}_{2}$ and $\mathrm{CO}$ which are stick onto grains. In a recent paper, Chapillon et al. (2011a), have observed the $\mathrm{H}_{2} \mathrm{D}^{+} 1_{1,1}-1_{1,0}$ transition with APEX in DM Tau and TW Hya. No detection was obtained but their upper limit was about 3 times better than the previous values. In their chemical analysis (using the gas-grain chemical model developed by Parise et collaborators 2011), they investigated several disk models (varying the density, temperature and outer radius), grain sizes (0.1, 1 and $10 \mu \mathrm{m})$, CO abundances $\left(10^{-4}, 10^{-5}\right.$ and $\left.10^{-6}\right)$ and rate of cosmic rays ionization $\left(10^{-17}, 3.10^{-17}\right.$ and $\left.10^{-16} \mathrm{~s}^{-1}\right)$. Their data only firmly exclude the case with both a low $\mathrm{CO}$ abundance and small grains. If the $\mathrm{CO}$ abundance is $\geqslant 10^{-5}$, any grain size can fit the data. This study clearly shows that $\mathrm{H}_{2} \mathrm{D}^{+}$is harder to detect than expected and that a significant sensitivity improvement is also needed to get constraining results on the physics and chemistry of the cold depleted mid-plane of TTauri disks.

\subsection{Impacts of the Dust Properties on Gas Phase Chemistry}

The dust is a major actor in these environments through several aspects and all chemical models are severely limited in their treatment of the influence of dust on the chemistry. The photoelectric effect on grains can affect the gas temperature, especially in the upper layers if the dust has already settled towards the disk mid-plane. Grain growth and dust settling directly affect the dust opacity, influencing the penetration of radiation through the medium and its thermal balance. Grain growth also affects the grain surface chemistry, by reducing the available surface area (per unit mass) but can also change the thermal balance in discs because the dust temperature is expected to depend on grain size. This has never been investigated by astro-chemical models and may significantly influence the gas disk structure because of the existence of important vertical and radial temperature gradients in disks, associated with strong grain growth. From a CO analysis of PdBI data obtained on the disks of CQ Tau and MWC758, Chapillon et al. (2008), reported a strong $\mathrm{CO}$ depletion $(\sim 100)$ which cannot be explained by depletion on 
grains since the disks are warm $(\sim 30-100 \mathrm{~K}$ at $100 \mathrm{AU})$. Interestingly, they suggest that CO may have been removed from the gas phase during the cold pre-stellar phase by adsorption on small grains. During the warmer proto-stellar phase, grain growth also occurs and CO may stay locked on larger grains which remain colder than small grains during reheating.

\subsubsection{Gas-to-Dust Ratio}

Dust settling along the mid-plane implies that the gas-to-dust ratio changes with the altitude in the disk. Recent observations of dust disks also suggest that the gas-to-dust ratio changes with radius. Indeed, Guilloteau et al. (2011) have reported the first evidences for radial segregation on grain size in several disks observed with the IRAM array at 2.8 and $1.3 \mathrm{~mm}$. They found that large grains are preferentially located in inner disks (within $R<50-70 \mathrm{AU}$ ) while the dust spectral index in the outer part of the disks suggest that these grains are small. As an example, Fig.4 presents the dust spectral index versus radius in the DL Tau disk. Such a result has lots of implications on the disk physics, it implies in particular that the gas-to-dust ratio and extinction curve in the UV are changing with radius. Future chemical models will have to take into account such changes in order to properly reproduce the physical conditions (e.g. UV illumination and penetration) in disks.

Measuring the gas-to-dust ratio is a difficult task both from the modeling and observational points of view. Following their CO interferometric study of the CQ Tau disk (Chapillon et al. (2008), Chapillon et al. (2010)) have performed using APEX a deep

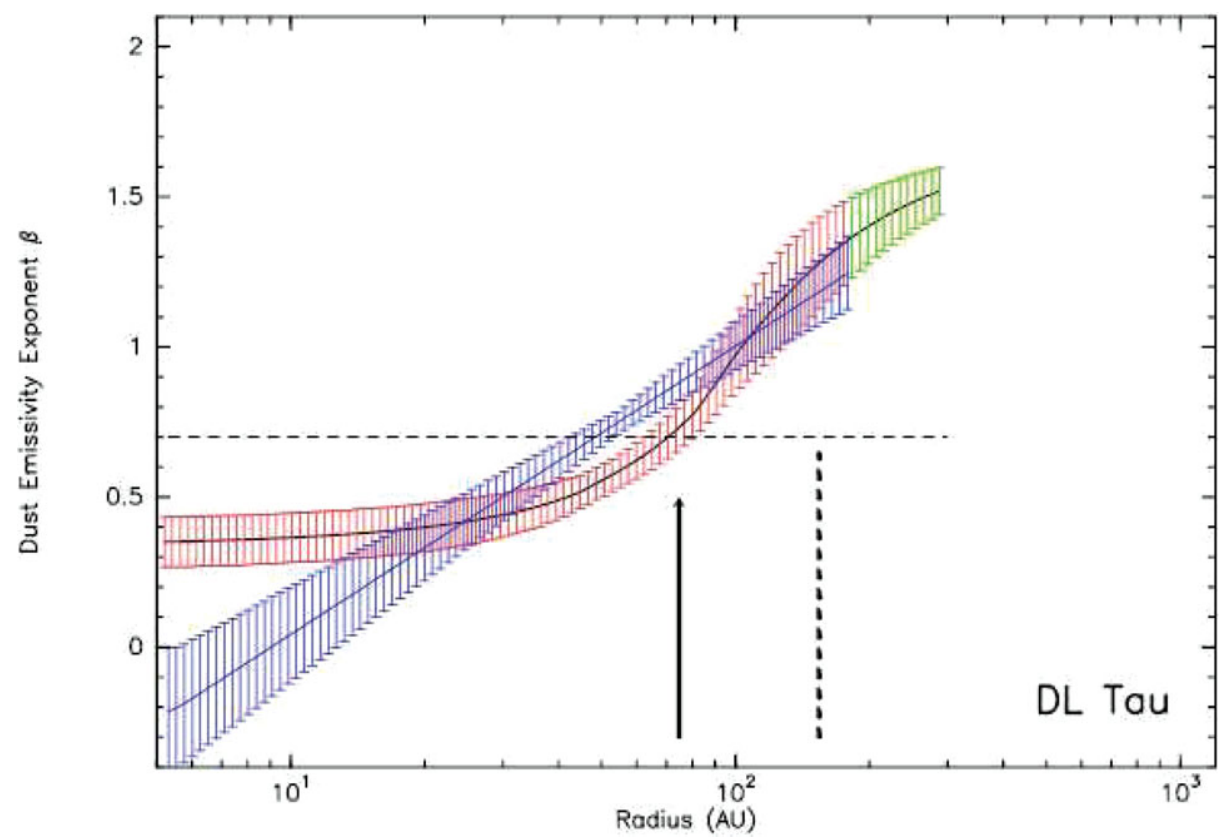

Figure 4. From Guilloteau et al. (2011). Derived dust emissivity (spectral index) versus radius in the dust disk surrounding the TTauri DL Tau. The spectral index has been derived from the resolved mm observations obtained with the IRAM array. There is a clear change of the dust properties within the radius. In the inner disk $(\mathrm{R}<50-60 \mathrm{AU})$, the dust is dominated by large particles with a $\beta$ value of $\sim 0.5$ while in the outer disk the dust appears ISM-like with a $\beta$ around $\sim 1.7$. Red and Green curves: two functionalities have been checked for the dust radial dependencies and the trend is the same in both cases. 
search for CI but failed to detect it. Their goal was to quantify the reservoir of Carbon. Using the PDR Meudon code, they simultaneously modeled the CO, CI and CII column densities and compared the result to the observed column densities of CO and CI (upper limit). Their study strongly suggests that only a low gas-to-dust ratio $(\lesssim 10)$ in presence of a weak UV field can explain the presence of $\mathrm{CO}$ and the non detection of CI. Therefore CQ Tau would be a disk in a transition phase between a proto-planetary gas-rich disk and a gas-free debris disk. Even though many unknowns remain, such a method seems promising to quantify the gas-to-dust ratio in disks where the physical structure is basically understood from resolved interferometric observations.

\subsubsection{Importance of Gas-Grains Interactions revealed by Sulfur Chemistry}

Protoplanetary disks are particular because they present strong density and temperature gradients on short spatial scales. For example, at 100 AU, the mid-plane temperature is expected to be around $7-12 \mathrm{~K}$ while 3 scale-heights above (about $30 \mathrm{AU}$ ), the temperature should be warm enough to allow the presence of a molecular layer. The chemical coupling between gas and dust through grain surface reactions may be a major ingredient of disk chemistry, this being emphasized by the presence of vertical turbulence since many molecules can remain trapped onto grains in the mid-plane.

In a recent paper, Dutrey et al. (2011) made a deep search for $\mathrm{SO}$ and $\mathrm{H}_{2} \mathrm{~S}$ using the 30-m radiotelescope in the disks surrounding GO Tau, DM Tau, LkCa15 and MWC480. They failed to detect these molecules but they analyzed the data together with the existing CS observations (obtained with IRAM facilities, CID project). They compared the molecular column densities derived from the observations with chemical predictions made using Nautilus (Hersant et al. (2009)), a gas-grain chemistry code dedicated to disks. For each disk, the density and temperature profiles have been taken from previous analysis (e.g. Pietu et al. (2007)). Fig.5 presents the ratio of the observed molecular densities (upper limits only for $\mathrm{SO}$ and $\mathrm{H}_{2} \mathrm{~S}$ ) to predicted column densities. Their model reproduces the $\mathrm{SO}$ and $\mathrm{CS}$ column densities reasonably well, but fails to match the upper limits obtained on $\mathrm{H}_{2} \mathrm{~S}$ by at least one order of magnitude. This suggests that at the high

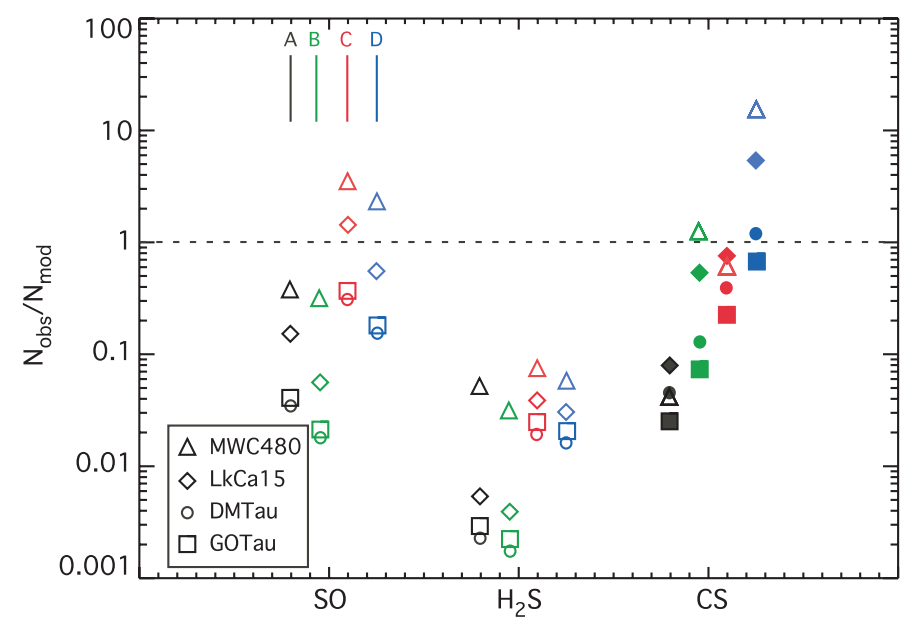

Figure 5. From Dutrey et al. (2011), CID paper V. Sulfur bearing observations with the IRAM 30-m radiotelescope $\left(\mathrm{SO}, \mathrm{H}_{2} \mathrm{~S}\right)$ and $\mathrm{PdBI}(\mathrm{CS})$. Constraining upper limits have been obtained on $\mathrm{SO}$ and $\mathrm{H}_{2} \mathrm{~S}$ allowing us to make a quantitative analysis of the Sulfur-bearing molecules in four disks. The observations have been analyzed using the chemical code Nautilus (Hersant et al. (2009)). 
densities and low temperatures encountered around disk mid-planes, $\mathrm{H}_{2} \mathrm{~S}$ remains locked onto the grain surfaces where it reacts to form other species preventing desorption of $\mathrm{H}_{2} \mathrm{~S}$. Indeed, some recent experiments by Garozzo et al. (2010) have shown that $\mathrm{H}_{2} \mathrm{~S}$ on grains is easily destroyed by cosmic rays and lead to the formation of $\mathrm{C}_{2} \mathrm{~S}, \mathrm{SO}_{2}$ and OCS on grains. These studies also suggest that most of the sulphur may be in the form of a sulphur rich residuum, which could be polymers of sulfur or amorphous aggregates of sulfur (Wakelam et al. (2004)). The associated grain surface reactions are not yet incorporated in chemical models.

These recent results emphasize the need of grain surface reactions in astro-chemical models in presence of high density and low temperature associated to a UV photon source, as it is the case in protoplanetary disks.

\section{Open Questions}

The detection rate of new molecules has not significantly improved since 2000 but with the advent of ALMA, the observation of chemistry in circumstellar disks is beginning a new era. However, there are some trends which appear from the recent observational results. Among them, it is important to mention:

- Multi-line, multi-isotopologue CO interferometric studies allow observers and modelers to retrieve the basic density and temperature structures of disks. This method will become more and more accurate with ALMA.

- Both $\mathrm{CO}, \mathrm{CN}, \mathrm{HCN}$ and $\mathrm{CCH}$ molecular studies apparently reveal very low temperature in the gas phase in several TTauri disks. These observations suggest that the molecular layer is at least partly colder than predicted and that there are some ingredients still missing in our understanding of the disk physics and/or chemistry. More accurate observational data will refine these studies.

- Dust is one of the major agent shaping the disk physics and chemistry. In particular, it plays a fundamental role to control the UV disk illumination and the temperature. It also strongly influences the chemistry since a significant part of the disk is at a temperature which is below the freeze out temperature of most molecules. Therefore chemical reactions on grain surface need to be incorporated in chemical models.

- Deriving the gas-to-dust ratio remains one of the most difficult task. This would be even more complex than current models suggest because recent dust observations reveal that the gas-to-dust ratio also changes radially.

- Finally, several upper limits such as that obtained on $\mathrm{H}_{2} \mathrm{D}^{+}$suggest that ALMA observations of disks may not be so easy and will require an adequate observational strategy.

\section{Acknowledgements}

I would like to acknowledge all CID members for a very fruitful collaboration, in particular S.Guilloteau, E.Chapillon, F.Hersant, D.Semenov, V.Pietu and T.Henning. I also thank all the KIDA team (http://kida.obs.u-bordeaux1.fr/) and V.Wakelam for providing chemical reactions as accurate as possible for astrophysics.

\section{References}

Aikawa Y. 2007 A $\& A, 656$, L93

D'Alessio P., Calvet N., Hartmann L., Lizano S., \& Cantó, J. 1999 ApJ, 527, 893

Chapillon E., Guilloteau S., Dutrey A., \& Piétu V. 2008 A\&A, 488, 565

Chapillon E., Parise B., Guilloteau S., Dutrey A., \& Wakelam V. 2010 A\&SA, 520, 61 
Chapillon E., Parise B., Guilloteau S., \& Du F. 2011 A\&A, in press

Chapillon E., Guilloteau S., Dutrey A., Pietu V., \& Guelin M. 2011 A $\& A$, in press

Chiang E. I. \& Goldreich P. 1997 ApJ, 490, 368

Dartois E., Dutrey A., \& Guilloteau S. 2003 A\&A, 399, 773

Dutrey, A. Guilloteau S., \& Guelin M. 1997 A\&A, 317, L55

Dutrey A., Guilloteau S., \& Ho P. 2007 Protostars and Planets V, Reipurth B., Jewitt D. \&6 Keil K. Eds, 495

Dutrey A., Henning T., Guilloteau S., Semenov D., Piétu V., Schreyer K., Bacmann A., Launhardt R., Pety J., \& Gueth F. 2007 A $\& A, 464,615$

Dutrey A., Guilloteau S., Piétu V., Chapillon E., Gueth F., Henning T., Launhardt R., Pavlyuchenkov Y., Schreyer K., \& Semenov D. 2008 A $\& A$, 490, L15

Dutrey A., Wakelam V., Boehler Y., Guilloteau S., Hersant F., Semenov D., Chapillon E., Henning T., Piétu V., Launhardt R., Gueth F., \& Schreyer K.2011 A\&\&A, in press

Garozzo M., Fulvio D., Kanuchova Z., Palumbo M. E., \& Strazzulla D. 2010, A\&A, 509, A67

Guilloteau S., Piétu, V., Dutrey A., \& Guélin M. 2006 A $\& A, 448$, L5

Guilloteau S., Dutrey A., Piétu V., \& Boehler Y. 2011 A $\& A$, X,Y

Henning T., Semenov D., Guilloteau S., Dutrey A., Hersant F., Wakelam V., Chapillon E., Launhardt R., Piétu V., \& Schreyer K. 2010 ApJ, 714, 1511

Hersant F., Wakelam V., Dutrey A., Guilloteau S., \& Herbst E. 2009 A $\& A$, 493, L49

Öberg K. I., Linnartz H., Visser R., \& van Dishoeck E. F. 2009 ApJ, 693, 1209

Piétu V., Dutrey A., \& Guilloteau S. 2007 A\&A, 467, 163

Qi C., Wilner D. J., Calvet N., Bourke T. L., Blake G. A., Hogerheijde M. R., Ho P. T. P., \& Bergin E. 2008 ApJ, 636, L157

Qi C., Wilner D. J., Aikawa Y., Blake G. A., \& Hogerheijde M. R. 2006 ApJ, 681, 1396

Semenov D., Wiebe D., \& Henning, T. 2006 ApJ, 647, L57

Semenov D., Hersant F., Wakelam V., Dutrey A., Chapillon E., Guilloteau S, Henning T., Launhardt R., Piétu V., \& Schreyer K. 2010 A 6 A, 522, A42

Wakelam V., Caselli P., Ceccarelli C., Herbst E., \& Castets A. 2004 A\& A, 422, 159

\section{Discussion}

E. VAN Dishoeck: Can you comment on the current state of determining the outer radius of the gas disk and its importance on the analysis?

A.Dutrey: The accuracy on the determination of the gas outer radius depends, of course, on the sensitivity and angular resolution of the observations. As usual, the S/N ratio must be properly taken into account to provide relevant error bars on the measurement. As soon as the molecular disk is spectroscopically and spatially resolved, which is the case for many mm interferometric data (at least those from PdBI), the outer radius measurement is accurate enough to have a very limited influence on the other parameters (details can be found in Dutrey et al. (2007a), Pietu et al. (2007)). Moreover, there are a few examples of CO disks (e.g. DM Tau, Dartois et al. (2003)) which suggest that selective photo-dissociation can explain the different disk sizes observed in ${ }^{12} \mathrm{CO}$ and in ${ }^{13} \mathrm{CO}$ (the ${ }^{13} \mathrm{CO}$ disk being smaller). 